\title{
Reflets
}

Revue ontaroise d'intervention sociale et communautaire

\section{La collaboration Cochrane : un outil de travail et de recherche pour les spécialistes de la réadaptation}

\section{Lynn Casimiro et Lucie Brosseau}

Volume 8, numéro 1, printemps 2002

La réadaptation : son visage français en Ontario

URI : https://id.erudit.org/iderudit/026379ar

DOI : https://doi.org/10.7202/026379ar

Aller au sommaire du numéro

Éditeur(s)

Reflets : Revue ontaroise d'intervention sociale et communautaire

ISSN

1203-4576 (imprimé)

1712-8498 (numérique)

Découvrir la revue

Citer cet article

Casimiro, L. \& Brosseau, L. (2002). La collaboration Cochrane : un outil de travail et de recherche pour les spécialistes de la réadaptation. Reflets, 8(1), 151-155. https://doi.org/10.7202/026379ar

Tous droits réservés (C) Reflets : Revue ontaroise d'intervention sociale et communautaire, 2002
Ce document est protégé par la loi sur le droit d'auteur. L'utilisation des services d'Érudit (y compris la reproduction) est assujettie à sa politique d'utilisation que vous pouvez consulter en ligne.

https://apropos.erudit.org/fr/usagers/politique-dutilisation/ 


\section{La collaboration Cochrane : un outil de travail et de recherche pour les spécialistes de la réadaptation.}

\section{Lynn Casimiro}

Centre national de formation en santé

\section{Lucie Brosseau}

École des sciences de la réadaptation (physiothérapie), Université d'Ottawa

Le défi des professionnels de la réadaptation repose sur la nécessité d'examiner et de justifier l'efficacité des traitements qu'ils pratiquent. La Collaboration Cochrane (CC) est un des outils permettant de relever ce défi. Le présent document contient un bref historique du développement de la $\mathrm{CC}$, une brève explication de son usage et une description de la méthode pour mener adéquatement une analyse systématique des écrits "à la Cochrane ".

Le nom de l'outil Cochrane provient de son promoteur, Docteur Archie Cochrane. Ce médecin britannique chérissait un rêve professionnel à répercussions sociales longuement mûri. Il désirait que les écrits scientifiques liés au domaine de la santé soient plus utilisés et plus accessibles aux professionnels de la santé ayant ainsi des répercussions au niveau de la qualité des services de santé offerts aux prestataires. Six ans après son décès, en 1993, la CC fut fondée. Depuis, un site Internet [www.cochrane.org], consacré à la CC, fournit des renseignements pertinents aux professionnels de la santé et au public en général. 
En soi, la CC consiste en une organisation internationale sans but lucratif, composée de professionnels de la santé. Le but de la CC est de coordonner des revues exhaustives de la littérature pour ensuite préparer la synthèse des résultats de recherches de type randomisé portant sur un même sujet. L'information, qui s'appuie sur les données probantes, est rendue accessible via le répertoire Cochrane. La banque des données est remise à jour d'une façon continue et peut facilement être consultée par les clients, les cliniciens, les chercheurs et les éducateurs du domaine de la santé. L'outil est accessible via Internet ou sur disque compact. Les cédéroms de la banque de données peuvent être achetés à un prix modique. Le répertoire est également disponible dans la majorité des bibliothèques médicales canadiennes affiliées à une université ou à un établissement de santé.

Mais quelles sont les particularités de la banque de données Cochrane? Il s'agit d'un répertoire électronique qui rassemble plus de 1,200 recensions systématiques (Cochrane Database of Systematic Reviews [CDSR]). De plus, le répertoire Cochrane comprend d'autres banques de données spécialisées. Par exemple :

- Les résumés de recensions portant sur les effets des interventions cliniques (Database of Abstracts of Reviews of Effectiveness [DARE]);

- Un registre répertoriant plus de 250,798 références liées à des essais cliniques randomisés (Cochrane Controlled Trials Register [CCTR]);

- Une liste bibliographique de plus de 1017 études traitant de sujets méthodologiques (Cochrane Review Methodology Database [RMD]).

Une particularité du répertoire Cochrane est sa classification des données en fonction du type d'intervention ou d'une discipline plutôt qu'en fonction d'une affection spécifique. À titre d'exemple, il existe des champs de recherche pour les infirmières, les médecins de première ligne et les spécialistes du domaine de la gériatrie. Des experts internationaux et des membres du public sont regroupés selon les champs de recherche pour veiller à la création de banques de données pertinentes. Notons que les domaines de 
la réadaptation et des thérapies connexes sont coordonnés dans les Pays-Bas.

La CC cible particulièrement les chercheurs en offrant un bilan objectif des connaissances et des faits scientifiques portant sur l'effet d'une intervention d'intérêt. Elle propose également des pistes de recherche à partir des résultats des recensions systématiques existantes. La CC appui également les chercheurs désireux de participer aux revues systématiques de la littérature selon la méthodologie particulière de Cochrane.Voici une brève description des étapes à suivre. Des explications plus détaillées peuvent être obtenues sur le site web de la CC (www.cochrane.org).

Premièrement, un protocole doit être soumis puis accepté par le coordonnateur de la CC associé au champ de recherche ciblé. Deuxièmement, la recension systématique Cochrane proprement dite doit être menée comme suit.

1- La formulation du problème : Le chercheur devra choisir un sujet portant sur l'efficacité d'une intervention spécifique en réadaptation.

2- Localisation et sélection des études : Une stratégie pour effectuer la revue de la littérature doit être élaborée. Plusieurs sources informatisées peuvent être utilisées incluant " MEDLINE ", "EMBASE ", le CCTR, les index et les bases de données, la bibliographie des textes importants ainsi que les comptes rendus des conférences. Les critères d'inclusion et d'exclusion doivent être bien documentés et suivre les recommandations de la CC. D'habitude, seules les études randomisées ou avec un groupe témoin peuvent être incluses. $\mathrm{Au}$ moins deux examinateurs indépendants doivent évaluer la pertinence des références identifiées. La recension systématique devra comprendre une description des études retenues ainsi que celles rejetées.

3- L'évaluation critique des études : La qualité méthodologique des essais cliniques randomisés devra être évaluée à l'aide d'une grille-synthèse validée (Jaddad 1996). L'évaluation de la qualité des études se rapporte à la méthode de randomisation des sujets, à la présence de devis à double insu 
et au recensement des désistements au cours de la collecte des données.

4- La collecte de données : Un formulaire d'extraction de données devra être conçu spécifiquement pour l'évaluation des études retenues. Le formulaire doit inclure des informations au sujet de la grandeur de l'échantillon, de l'âge moyen des sujets, de l'écart type des différents groupes. Il doit aussi contenir les caractéristiques spécifiques de l'intervention ainsi que les résultats obtenus. Lors de l'extraction des données brutes, les chercheurs devront inclure une section permettant de compiler les résultats de chacun des groupes traités (groupes témoins versus expérimentaux) selon chaque mesure de résultats. La concordance d'au moins deux réviseurs devra être obtenue au niveau de la vérification des données compilées avant même d'entreprendre l'analyse des données.

5- Analyse et présentation des données : Les données extraites sont analysées à l'aide d'un logiciel spécifique "RevMan » conçu par un épidémiologiste de la Collaboration Cochrane (Clarke 1999). Différentes méthodes statistiques sont utilisées en fonction du type de données recueillies. Le chercheur peut gratuitement télé-décharger la version la plus récente du logiciel « RevMan » à partir du site Internet de la collaboration Cochrane. Ce logiciel produira des résultats compilés selon une forme graphique conçue par la collaboration Cochrane.

6- Interprétation des résultats : Les résultats doivent être discutés à la lumière des pratiques courantes et de ce qui est connu au sujet de l'intervention à l'étude. Les problèmes méthodologiques des études répertoriées devront y être présentés.

Il est à noter que la $\mathrm{CC}$ exige des chercheurs d'ajouter leurs recensions systématiques publiées dans le CCTR. La CC produit à tous les trois mois une nouvelle édition de la bibliothèque Cochrane permettant l'ajout de nouvelles recensions. 
D'une part, mener une recension systématique Cochrane portant sur les effets d'une intervention en réadaptation constitue une façon rapide et efficace d'obtenir une vision globale du niveau des connaissances scientifiques actuelles. D'autre part, une revue systématique peut stimuler et inciter les chercheurs du domaine de la réadaptation à identifier les domaines de la recherche clinique dont les connaissances scientifiques sont déficientes. En effet, l'outil de travail et de recherche Cochrane peut s'avérer utile pour les professionnels de la réadaptation en les aidant à faire avancer les connaissances et probablement leur domaine d'expertise.

\section{Références}

CLAR KE, M.,AD. OXMAN (1999). «Cochrane Reviewers' Handbook 4.0 », Review Manager(RevMan - logiciel), Oxford England, The Cochrane Library.

JADAD AR, RA. MOORE, D. Caroll, et al. (1996). "Assessing the quality of reports of randomized trial : is blinding necessary? ", Control Clinical Trials, 17, 1-12. 